\title{
Die kollisionsrechtliche Behandlung von Treuhandbeteiligungen
}

\author{
Anmerkung zum Urteil des EuGH vom 3. Oktober 2019 \\ (Rs. C-272/18, VKI./.TVP)
}

Dr. Anja Sophia Schwemmer, Berlin*

Inhaltsübersicht $\quad$ ZGR 2020, 1078-1093

I. Einleitung . . . . . . . . . . . . . . . . . . . . . . . . . . . . . 1080

II. Sachverhalt der Rechtssache VKI./.TVP . . . . . . . . . . . . . . . . . . . . . 1081

III. Die Figur des Quasi-Gesellschafters im deutschen Gesellschaftsrecht . . . . . . 1081

1. Ausgestaltung von Treuhandbeteiligungen an deutschen Publikumspersonengesellschaften . . . . . . . . . . . . . . . . . 1082

2. Richterliche Rechtsfortbildung . . . . . . . . . . . . . . . . . . 1082

IV. Kollisionsrechtliche Behandlung des Treuhandvertrags . . . . . . . . . . . . . 1084

1. Gespaltene Anknüpfung . . . . . . . . . . . . . . . . . . . . . . . 1084

2. Kriterien zur Abgrenzung zwischen Gesellschafts- und Vertragsstatut . . 1086

3. Verbraucherschützende Sonderanknüpfung . . . . . . . . . . . . . . . . . . . 1088

4. Rechtsmissbräuchlichkeit der Rechtswahlklausel . . . . . . . . . . . . . 1089

V. Konsequenzen der Entscheidung für bestehende Treuhandbeteiligungen . . . 1090

1. Rechtsunsicherheit über das auf den Treuhandvertrag anwendbare Recht 1091

2. Friktionen zwischen Gesellschafts- und Treuhandvertragsstatut . . . . . 1091

VI. Zusammenfassende Thesen . . . . . . . . . . . . . . . . . . . . . . . . . . . . . 1092

In seinem Urteil vom 3. Oktober 2019 (Rs. C-272/18 - VKI./.TVP) hatte der EuGH sich mit der bislang kaum diskutierten, aber praktisch relevanten Frage zu beschäftigen, welches Recht auf Treuhandbeteiligungen anzuwenden ist. Die entscheidende Weichenstellung war dabei die Frage der kollisionsrechtlichen Qualifikation. Neben der Einordnung als Schuldvertrag kam anfgrund der Gleichstellung der Trengeber als sog. „Quasi-Gesellschafter" mit den Direktkommanditisten eine Zuordnung zum Gesellschaftsstatut in Betracht. Der EuGH nabm jedoch in seinem Urteil nicht den gesamten Treuhandvertrag in den Blick, sondern beschäftigte sich nur mit einem Teil der darin enthaltenen Regelungen, die er schuldrechtlich qualifizierte. Das anwendbare Recht richte sich insoweit nach den Regelungen der Rom I-Verordnung. Ferner entschied der EuGH, dass bei einem Vertrieb an Verbraucher die Sonderanknüpfung des Art. 6 Rom I-VO zur Anwendung kommt. Für Verbraucherverträge hatte der EuGH bereits zuvor entschieden, dass Rechtswablklauseln, die nicht über ibre beschränkte Wirkung im Sinne des Art. 6 Abs. 2 Rom I-VO aufklären, missbräuchlich und daher nichtig sind. Danach kommt im Ergebnis das Aufenthaltsrecht des Verbrauchers zur Anwendung. Die nachfolgende Besprechung analysiert das Urteil unter Berücksich-

* Die Verf. ist Rechtsanwältin/Steuerberaterin in Berlin und Habilitandin am Lehrstuhl von Prof. Dr. Marc-Philippe Weller an der Universität Heidelberg. 
tigung der Rechtsfigur des Quasi-Gesellschafters und zeigt dabei die dogmatischen Schwächen in der Argumentation des EuGH aus kollisionsrechtlicher Sicht sowie die zentralen Fragen auf, die der Gerichtshof offen gelassen hat. Schließlich werden die Konsequenzen beleuchtet, die sich aus einem möglichen Auseinanderfallen von Gesellschaftsstatut und Treubandvertragstatut ergeben.

In its ruling of 3 October 2019 (Case C-272/18 - VKI./.TVP), the ECJ had to deal with the question of the law applicable to fiduciary partnership interests, which has hardly been discussed so far but is of practical relevance. The decisive point of departure was the characterization under conflict of laws. Due to the equal legal status of the fiduciary partners (so called "Quasi-Gesellschafter" under German law) and the direct limited partners, it could be argued that the trust agreement should fall under the applicable company law regime rather than being classified as an ordinary contract. In its ruling, the ECJ did not answer this question for the entire trust agreement, but dealt solely with some of the clauses, which were qualified as contractual obligations falling under the Rome I Regulation. Consequently, the ECJ decided that for cases involving consumers as holders of the fiduciary partnership interest the special provision of Art. 6 Rome I Regulation applies. For consumer contracts, however, the ECJ had already previously ruled that choice of law clauses which do not clarify their limited effect as specified in Art. 6(2) Rome I Regulation are unfair and therefore void. As a result, the consumer's right of residence applies. The following article analyzes the judgment in consideration of the concept of the "Quasi-Gesellschafter" under German company law. It shows the dogmatic weaknesses in the ECJ's reasoning from a conflict-of-laws perspective and points to the crucial questions the court has left open. Finally, the consequences arising from a possible discrepancy between the law applicable to the trust agreement and the company law regime will be lined out.

\section{Leitsatz}

1. Art. 1 Abs. 2 Buchst. e des am 19.6.1980 in Rom zur Unterzeichnung aufgelegten Übereinkommens über das auf vertragliche Schuldverhältnisse anzuwendende Recht und Art. 1 Abs. 2 Buchst. f der VO (EG) Nr. 593/2008 des Europäischen Parlaments und des Rates vom 17.6.2008 über das auf vertragliche Schuldverhältnisse anzuwendende Recht (Rom I) sind dahin auszulegen, dass vertragliche Pflichten wie die im Ausgangsverfahren in Rede stehenden, die ihren Ursprung in einem Treuhandvertrag über die Verwaltung einer Beteiligung an einer Kommanditgesellschaft haben, nicht vom Anwendungsbereich des Übereinkommens und der Verordnung ausgenommen sind.

2. Art. 5 Abs. 4 Buchst. $b$ des Übereinkommens über das auf vertragliche Schuldverhältnisse anzuwendende Recht und Art. 6 Abs. 4 Buchst. a der VO Nr. 593/2008 sind dahin auszulegen, dass ein Treuhandvertrag, aufgrund dessen die dem Verbraucher geschuldeten Dienstleistungen in dem Staat, in dem er seinen gewöhnlichen Aufenthalt hat, vom Gebiet eines anderen Staates aus, d.h. aus der Ferne, zu erbringen sind, nicht unter den in diesen Bestimmungen vorgesehenen Ausschluss fällt.

3. Art. 3 Abs. 1 der RL 93/13/EWG des Rates vom 5.4.1993 über missbräuchliche Klauseln in Verbraucherverträgen ist dahin auszulegen, dass eine in einem zwischen einem Unternehmer und einem Verbraucher abgeschlossenen Treuhandvertrag über die Verwaltung einer Kommanditbeteiligung, wie sie im Ausgangsverfahren in Rede stehen, enthaltene Klausel, die nicht im Einzelnen 
ausgehandelt wurde und nach der das Recht des Sitzmitgliedstaats der Kommanditgesellschaft anwendbar ist, missbräuchlich im Sinne der genannten Bestimmung ist, wenn sie den Verbraucher in die Irre führt, indem sie ihm den Eindruck vermittelt, auf den Vertrag sei nur das Recht dieses Mitgliedstaats anzuwenden, ohne ihn darüber zu unterrichten, dass er nach Art. 5 Abs. 2 des Übereinkommens über das auf vertragliche Schuldverhältnisse anzuwendende Recht und Art. 6 Abs. 2 der Verordnung Nr. 593/2008 auch den Schutz der zwingenden Bestimmungen des nationalen Rechts genießt, das ohne diese Klausel anzuwenden wäre.

\section{Einleitung}

Anleger beteiligen sich an Fonds in der Form von Publikumspersonengesellschaften häufig nicht unmittelbar als Gesellschafter, sondern über Treuhandbeteiligungen. Auch die neuen blockchainbasierten Anlageformen, sog. Equity Token $^{1}$, die zuletzt als Möglichkeit der Kapitalaufnahme an Bedeutung gewonnen haben, sind teilweise als Treuhandbeteiligungen strukturiert. ${ }^{2}$ Treuhandund Gesellschaftsverträge sind dabei typischerweise so eng verzahnt, dass die Rechtsprechung den Treugeber als sog. "Quasi-Gesellschafter" qualifiziert, der im Innenverhältnis einem unmittelbar beteiligten Gesellschafter nicht nur wirtschaftlich, sondern auch rechtlich gleichgestellt ist.

Oft werden solche Treuhandbeteiligungen auch grenzüberschreitendend an (Privat-)anleger im Ausland vertrieben. Dabei liegt es nahe, den Treuhandvertrag dem Gesellschaftsstatut der jeweiligen Fondsgesellschaft zu unterwerfen und so Friktionen zu vermeiden, beispielsweise bei den praktisch bedeutsamen Klagen auf Rückabwicklung der Beteiligung. ${ }^{3}$ Um diesen kollisionsrechtlichen Gleichlauf sicherzustellen, enthalten die Treuhandverträge in aller Regel Rechtswahlklauseln zugunsten des Heimatrechts der Fondsgesellschaft. Mit einer solchen Rechtswahlklausel in einem Treuhandvertrag hatte sich der EuGH in der Rechtssache VKI./.TVP zu beschäftigen. Dem vorgeschaltet war die Frage nach der kollisionsrechtlichen Qualifikation des Treuhandvertrages. Die Abgrenzung zwischen Vertrags- und Gesellschaftsstatut war deshalb von zentraler Bedeutung, weil im internationalen Schuldvertragsrecht nach der Rom I-VO anders als im internationalen Gesellschaftsrecht einerseits eine Rechtswahl zulässig ist und andererseits mit Art. 6 Rom I-VO eine Sonderanknüpfung zum Schutz von Verbrauchern existiert.

1 Vgl. zum Begriff z. B. Hanten/Sacarcelik, RdF 2019, 124, 125.

2 Hanten/Sacarcelik, RdF 2019, 124, 130; Behme/Zickgraf, ZfPW 2019, 66.

3 Dafür z.B. KrÜger/Eichwald, EWiR 2020, 37. 
Im Folgenden wird zunächst die Ausgestaltung von Treuhandbeteiligungen und die Rechtsfigur des sog. Quasi-Gesellschafters erläutert (III.). Vor diesem Hintergrund wird die Entscheidung des EuGH zusammengefasst und aus kollisionsrechtlicher Sicht kritisch gewürdigt (IV.). Schließlich werden die Konsequenzen der Entscheidung insbesondere für bestehende Treuhandbeteiligungen beleuchtet (V.).

\section{Sachverbalt der Rechtssache VKI./.TVP}

Der Entscheidung in der Rechtssache VKI./.TVP lag ein Vertragswerk eines Publikumsfonds in Form einer deutschen Kommanditgesellschaft zugrunde. An der Fondsgesellschaft beteiligten sich Privatanleger als Treugeber über TVP als Treuhandkommanditistin. Durch die eng verzahnten Regelungen des Gesellschafts- und Treuhandvertrags wurde den Treugebern die Rechtsstellung eines sog. Quasi-Gesellschafters eingeräumt. Der Fonds wurde durch eine Tochtergesellschaft der Fondsinitiatorin auch in Österreich vertrieben. $\mathrm{Zu}$ diesem Zweck wurde eine Webseite mit der Adressendung „.at“ betrieben. In dem Treuhandvertrag, auf dessen Grundlage die TVP die Beteiligungen der Anleger hielt und verwaltete, war eine Rechtswahlklausel zugunsten des deutschen Rechts enthalten. Gegen diese Rechtswahlklausel wandte sich der österreichische Verbraucherverband VKI mit einer Unterlassungsklage.

\section{Die Figur des Quasi-Gesellschafters im deutschen Gesellschaftsrecht}

Sowohl der EuGH als auch die ersten Kommentatoren ${ }^{4}$ des Urteils in der Literatur beschäftigen sich trotz des entsprechenden Vortrags der TVP im Verfahren nicht näher mit der durch das Vertragswerk aus Treuhand- und Gesellschaftsvertrag begründeten Rechtsstellung der Treugeber als Quasi-Gesellschafter. Jedoch ergibt sich die Komplexität der kollisionsrechtlichen Fragestellung aus dieser Rechtsfigur: Der qualifizierte Treuhandvertrag ist eben gerade kein Vertrag, der einen Gesellschaftsanteil bloß zum Gegenstand hat, wie beispielsweise ein Anteilskaufvertrag. ${ }^{5}$ Vielmehr wird durch ihn (im $\mathrm{Zu}-$ sammenspiel mit dem Gesellschaftsvertrag der Fondsgesellschaft) die QuasiGesellschafterstellung der Treugeber erst begründet.

4 Reuter, WuB 2020, 107, 110; Mankowski, LMK 2019, 422737.

5 So aber Mankowski, LMK 2019, 422737; Reuter, WuB 2020, 107, 110; vgl. auch GA Øe, Schlussanträge v. 5.9.2019, Rs. C-272/18 - VKI./.TVP, Rdn. 49. 


\section{Ausgestaltung von Treubandbeteiligungen an deutschen Publikumspersonengesellschaften}

Bei Treuhandbeteiligungen an Fondsgesellschaften schließt ein Treuhandkommanditist mit einer Vielzahl von Anlegern Treuhandverträge. Der Treuhänder hält danach seinen einheitlichen Gesellschaftsanteil zwar im eigenen Namen, aber für Rechnung der Anleger. Unter anderem aus steuerlichen Gründen ${ }^{6}$ werden die Treugeber einem unmittelbaren Gesellschafter regelmäßig nicht nur wirtschaftlich gleichgestellt, sondern wird auch ihre Rechtsstellung weitgehend derjenigen der Direktkommanditisten angenähert. $\mathrm{Zu}$ diesem Zweck wird dem Treugeber die Möglichkeit verschafft, bestimmte Gesellschafterrechte selbst auszuüben (sog. qualifizierte Treuhand). ${ }^{7}$ Insoweit finden sich in der Praxis verschiedene Gestaltungsmodelle: ${ }^{8}$

- Teils wird den Treugebern nur im Treuhandvertrag Vollmacht zur Ausübung bestimmter Gesellschafterrechte der Treuhänderin (beispielsweise des Stimmrechts) erteilt.

- Oft - und so auch im Vorlagefall - weist aber schon der Gesellschaftsvertrag der Fondsgesellschaft den Treugebern bestimmte originäre Rechte zu, insbesondere das Stimmrecht und das Teilnahmerecht in der Gesellschafterversammlung. Diese gesellschaftsvertraglichen Regelungen werden meist durch korrespondierende Klauseln im Treuhandvertrag inkorporiert. So entsteht eine enge Verzahnung der beiden Verträge zu einem ineinandergreifenden Regelwerk.

\section{Richterliche Rechtsfortbildung}

In der Rechtsprechung des BGH hat sich für qualifizierte Treuhandbeteiligungen die Figur des sog. „Quasi-Gesellschafters“ herausgebildet. Hierunter ver-

6 In der Regel soll der Treugeber aus steuerlicher Sicht Mitunternehmer i.S.d. $\$ 15$ Abs. 1 Satz 1 Nr. 2 EStG werden. Dies setzt voraus, dass der Treugeber durch eine vermögensmäßige Beteiligung an den Gewinnen und Verlusten der Gesellschaft ein Mitunternehmerrisiko trägt sowie Mitunternehmerinitiative entfalten kann. Letzteres setzt regelmäßig Kontroll- und Teilhaberechte voraus, die denen eines Kommanditisten entsprechen. Allerdings wurden die Möglichkeiten der Verlustzuweisung über derartige Mitunternehmerschaften erheblich eingeschränkt, so dass die steuerlichen Gründe für die Gleichstellung von Treugebern und Direktkommanditisten inzwischen an Bedeutung verloren haben.

7 Vgl. zur Differenzierung zwischen echter und qualifizierter Treuhand Jahnke, Der Quasi-Gesellschafter, 2019, S. 19, 33; WiedEMANN, ZIP 2012, 1786, 1787; ähnlich CASPER, in: Staub, Großkomm. z. HGB, 5. Aufl., 2015, $\$ 161 \mathrm{Rdn} .239 \mathrm{ff}$.

8 Asmus/Mark wardt, ZIP 2012, 1581, 1583 ff; Jahnke, aaO (Fn. 7), S. 33 ff; Trinks, in: Behme/Fries/Stark, Versicherungsmechanismen im Recht, 2016, S. 149, $156 \mathrm{f}$. 
steht der BGH einen Treugeber, der im Innenverhältnis einem unmittelbar beteiligten Gesellschafter vollständig gleichgestellt ist. Durch die Verzahnung von Gesellschafts- und Treuhandvertrag sei der Treugeber in den Gesellschaftsverband einbezogen. Eine solche Quasi-Gesellschafterstellung ist nach der Rechtsprechung des BGH in der oben dargestellten zweiten Fallgruppe anzunehmen, d. h. wenn dem Treugeber im Gesellschaftsvertrag selbst originäre Gesellschafterrechte, insbesondere das Stimmrecht, eingeräumt werden. ${ }^{9}$

Diesem Quasi-Gesellschafter gesteht die Rechtsprechung dann - über die explizit vertraglich eingeräumten Rechte hinaus - zwingende gesetzliche Gesellschafterrechte wie ein Auskunftsrecht über die Mitgesellschafter und Treugeber ${ }^{10}$ oder den Aufwendungsersatzanspruch nach $\$ 110 \mathrm{HGB}^{11} \mathrm{zu}$. Darüber hinaus kann der Quasi-Gesellschafter bei Aufklärungsfehlern im Rahmen des Erwerbs der Treuhandbeteiligung oder bei Bestehen von Verbraucherwiderrufsrechten hinsichtlich des Erwerbsgeschäfts unmittelbar gegenüber der Fondsgesellschaft seine Beteiligung kündigen und sie auf Auszahlung des Auseinandersetzungsguthabens in Anspruch nehmen. ${ }^{12}$

Die rechtliche Gleichstellung der Quasi-Gesellschafter beschränkt sich aber nach Auffassung des BGH auf das Innenverhältnis. ${ }^{13}$ Nur insoweit stehe es den Beteiligten frei, ihre Rechtsbeziehungen durch freie vertragliche Regelung zu gestalten; ${ }^{14}$ sie könnten daher auch Dritte einem Gesellschafter gleichstellen. Im Verhältnis zu Dritten gilt die Gleichstellung hingegen nicht. Insbesondere eine Haftung im Außenverhältnis ist mit der Stellung als Quasi-Gesellschafter nicht verbunden. ${ }^{15}$

9 BGH, Urt. v. 5.2.2013, II ZR 134/11, NJW 2013, 2190; Urt. v. 11.10.2011, II ZR 242/09, NZG 2011/1432, Rdn. 16ff; Urt. v. 11.11.2008, XI ZR 468/07, NZG 2009, 57, Rdn. 19.

10 BGH, Urt. v. 5.2.2013, II ZR 134/11, NJW 2013, 2190; Urt. v. 16.12.2014, II ZR 277/13, ZIP 2015, 319.

11 BGH, Urt. v. 24.7.2012, II ZR 297/11, NJW 2013, 452, 455 f.

12 BGH, Urt. v. 20.1.2015, II ZR 444/13, NJW 2015, 1169, 1170 (außerordentliche Kündigung wegen Aufklärungsfehler); Urt. v. 2.7.2001, II ZR 304/00, NJW 2001, 2718 (Haustürwiderrufsrecht gegenüber der Fondsgesellschaft). Vgl. zusammenfassend TRINKs, aaO (Fn. 8), S. 149, 160 f.

13 Kritisch Trinks, aaO (Fn. 8), S. 149, $168 \mathrm{ff}$.

14 St. Rspr., vgl. nur BGH, Urt. v. 11.10.2011, II ZR 242/09, NZG 2011/1432, Rdn. 16ff; ausführlich hierzu Trinks, aaO (Fn. 8), S. 149, $168 \mathrm{ff}$.

15 BGH, Urt. v. 29.9.2015, II ZR 403/13, NJW 2015, 3789; Rdn. 20; Urt. v. 11.11.2008, XI ZR 468/07, NZG 2009, 57, Rdn. $21 \mathrm{f}$. 


\section{Kollisionsrechtliche Behandlung des Treubandvertrags}

Mit seiner ersten Vorlagefrage bat der österreichische OGH um Klärung, ob ein solcher Treuhandvertrag als rein schuldrechtlicher Vertrag unter den Anwendungsbereich der Rom I-VO fällt oder ob er gesellschaftsrechtlich zu qualifizieren ist und deshalb von der Bereichsausnahme des Art. 1 Abs. 2 lit. $f$ Rom I-VO für Fragen des Gesellschaftsrechts erfasst ist. Diese Frage stellte sich vor dem Hintergrund der beschriebenen engen Verzahnung zwischen dem Gesellschaftsvertrag der Fondsgesellschaft und dem Treuhandvertrag, durch die die Treugeber als Quasi-Gesellschafter in den Gesellschaftsverbund einbezogen wurden. Im Rahmen dieser Gestaltung verliert der Treuhandvertrag seinen rein schuldrechtlichen Charakter. Er wird Teil eines einheitlichen Vertragswerkes, das verbandsrechtliche Züge trägt. Auf der kollisionsrechtlichen Ebene wirft diese Verflechtung zu einem einheitlichen Vertragswerk die Frage nach der zutreffenden Qualifikation des Treuhandvertrags auf.

\section{Gespaltene Anknüpfung}

Der EuGH nimmt in seiner Entscheidung leider nicht umfassend zur Qualifikation eines solchen Treuhandvertrags Stellung, obwohl die Vorlagefrage in diese Richtung formuliert war. Vielmehr beschränkt sich der EuGH - wie bereits der Generalanwalt $\varnothing e$ in seinen Schlussanträgen ${ }^{16}$ - auf die Qualifikation bestimmter Klauseln des Treuhandvertrags. ${ }^{17}$ Diese sollen nach Auffassung des EuGH für sich genommen unter das Vertragsstatut fallen, da sie „nicht die Tragweite etwaiger Rechte und Pflichten, die die Treugeber nach dem anwendbaren Gesellschaftsrecht gegenüber der Kommanditgesellschaften haben, oder ihre etwaigen Verbindlichkeiten gegenüber Drittgläubigern der Gesellschaft" betreffen, sondern Fragen wie den Umfang der Haftung der Treuhänderin, den Erfüllungsort der Treuhandleistungen sowie das auf den Treuhandvertrag anwendbare Recht. ${ }^{18}$ Zwar führt Generalanwalt $\varnothing e$ in seinen Schlussanträgen aus, dass die - eben auch durch den Treuhandvertrag begründete - Rechtsstellung der Treugeber als Quasi-Gesellschafter durchaus dem Gesellschaftsstatut zuzuordnen sei; dies ändere aber nichts am schuldrechtlichen Charakter der übrigen Regelungen des Treuhandvertrags. ${ }^{19}$

16 GA ØЕ, Schlussanträge v. 5.9.2019, Rs. C-272/18 - VKI./.TVP, Rdn. 57.

17 EuGH, Urt. v. 3.10.2019, Rs. C-272/18 - VKI./.TVP, Rdn. 37.

18 EuGH, Urt. v. 3.10.2019, Rs. C-272/18 - VKI./.TVP, Rdn. 37, 39.

19 GA ØE, Schlussanträge v. 5.9.2019, Rs. C-272/18 - VKI./.TVP, Rdn. 56f; vgl. auch KLOCKE, DZWIR 2020, 61, 63. 
Diese Vorgehensweise ist methodisch fragwürdig. ${ }^{20}$ Indem der EuGH einzelne Abreden isoliert und ohne Rücksicht auf die den Vertrag prägenden Hauptbestandteile qualifiziert, macht das Gericht die gespaltene Anknüpfung von (Gesellschafts-)verträgen zum Regelfall. Dieser Weg widerspricht schon ganz grundsätzlich dem der Rom I-VO zugrundeliegenden Konzept eines einheitlichen objektiven ${ }^{21}$ Vertragsstatuts, nach dem alle einen Vertrag betreffenden Fragen anzuknüpfen sind..$^{22}$ Für eine solche kollisionsrechtlichen Vertragsspaltung (dépeçage $)^{23}$ besteht auch kein nachvollziehbarer Grund, wenn es sich wie hier bei den gesondert anzuknüpfenden Vertragsbestandteilen um Nebenabreden wie Rechtswahlklauseln oder Regelungen zum Erfüllungsort handelt, die den Charakter des Vertrages nicht prägen, sondern vielmehr notwendig auf die vertragscharakteristischen Abreden bezogen sind. Allenfalls bei echten typengemischten Verträgen mag eine solche gespaltene Anknüpfung sinnvoll sein.

Frühere Äußerungen des EuGH zur Auslegung der Ausschlussklausel nach Art. 1 Abs. 2 lit. f) Rom I-VO deuteten aber bereits in eine ähnliche Richtung. So ordnete der EuGH in der Rechtssache Kerr./.Postnov die Zahlungspflichten zweier Wohnungseigentümer gegenüber einer Wohnungseigentümergemeinschaft dem Schuldvertragsstatut zu, weil es sich insoweit nicht um die „organisatorischen“ Aspekte einer etwaigen Gesellschaft handele. ${ }^{24}$ Der EuGH scheint also auch hier davon auszugehen, dass Gesellschaftsverträge im Regelfall nicht einheitlich dem Gesellschaftsstatut unterfallen, sondern in Teilen dem Schuldvertragsstatut zuzuordnen sind. Zu Ende gedacht führt dieser Ansatz

20 Ablehnend auch Thomale, NZG 2020, 328, 332; KrÜger/Eichwald, EWiR 2020, 37. Dem EuGH uneingeschränkt zustimmend aber Mankowski, LMK 2019, 422737; CzAPLINSKI, jurisPR-IWR 6/2019 Anm. 1.

21 Die Parteien können im Rahmen ihrer Rechtswahl den Vertrag weiterhin verschiedenen Rechtsordnungen unterstellen (Art. 3 Abs. 1 Satz 3 Rom I-VO). Der Richter soll diese Möglichkeit aber nicht mehr haben.

22 Magnus, in: Staudinger, Internationales Vertragsrecht I, Neubearb. 2016, Einl Rom IVO Rdn. 74, Art. 4 Rom I-VO, Rdn. 9; Martiny, Münchener Komm. z. BGB, 7. Aufl., 2018, Art. 4 Rom I-VO, Rdn. 9; Leible/Lehmann, RIW 2008, 528, 536; a. A. ManKOwskI, FS Spellenberg, 2010, S. 261, $267 \mathrm{ff}$. Das EVÜ erlaubte hingegen noch in Art. 4 Abs. 1 Satz 2 EVÜ (und übereinstimmend Art. 28 Abs. 1 Satz 2 EGBGB a.F.) bei komplexen Vertragsverhältnisses eine ausnahmsweise Abspaltung einzelner Vertragsbestandteile. Vgl. dazu v. Hoffmann/Thorn, Internationales Privatrecht, 9. Aufl., 2007, $\$ 10$ Rdn. 38; Windmöller, Vertragsspaltung im Internationalen Privatrecht des EGBGB und des EGVVG, 2000.

23 Vgl. zum Begriff ausführlich WindmölleR, aaO (Fn. 22), S. 20 f; Mankowski, FS Spellenberg, S. 261, 262 begrenzt den Begriff der dépeçage auf die gespaltene Anknüpfung mehrerer Rechtsfragen trotz einheitlicher Qualifikation. Weiter z.B. v. HeIn, Münchener Komm. BGB, 7. Aufl., 2018, Einl. IPR, Rdn. 105 (Anwendung der Rechtsnormen verschiedener Staaten auf Teilaspekte eines Falles).

24 EuGH, Urt. v. 8. 5. 2019, Rs. C-25/18 - Kerr./.Postnov, Rdn. 33. Der EuGH wich insoweit von den Schlussanträgen der GA Кокотт v. 31.1.2019, dort Rdn. 60, ab. 
regelmäßig zu einer kollisionsrechtlichen dépeçage von Gesellschaftsverträgen, also einem Auseinanderreißen des Geflechts von eng aufeinander bezogenen Rechten, Pflichten und Nebenabreden. Dies verkennt aber den Zweck der Ausschlussklausel in Art. 1 Abs. 2 lit. f) Rom I-VO: ${ }^{25}$ Sie soll schließlich gerade gewährleisten, dass sämtliche das Gesellschaftsverhältnis betreffenden Fragen zur Vermeidung von Anpassungsproblemen einheitlich dem Gesellschaftsstatut unterworfen werden. ${ }^{26}$

\section{Kriterien zur Abgrenzung zwischen Gesellschafts- und Vertragsstatut}

Da der EuGH nur auf diejenigen Klauseln des Treuhandvertrags blickt, die für sich genommen schuldrechtlicher Natur sind, setzt er sich mit der Trennlinie zwischen dem Schuldvertragsstatut und dem Gesellschaftsstatut und der Rechtsfigur des Quasi-Gesellschafters kaum auseinander. Eine solche Auseinandersetzung wäre aber im Sinne der Rechtssicherheit im kollisionsrechtlichen Grenzgebiet zwischen Schuldrecht und Gesellschaftsrecht durchaus wünschenswert gewesen.

Dies gilt insbesondere deshalb, weil sich in der Rechtsprechung des EuGH eine von der ganz überwiegenden Auffassung in der deutschen Rechtsprechung und Literatur abweichende Grenzziehung abzuzeichnen scheint. Nach der noch zum EGBGB entwickelten Auffassung verläuft die Trennlinie zwischen Gesellschafts- und Schuldvertragsstatut entlang der Unterscheidung zwischen Innen- und Außengesellschaft. Kollisionsrechtlich maßgeblich ist vor allem die Frage, ob das Gesellschaftsverhältnis Rechtswirkungen über den Gesellschafterkreis hinaus nach außen entfaltet. ${ }^{27}$ Begründet wird diese Abgrenzung damit, dass Gesellschaftsverträge zum Zweck des Schutzes gesellschaftsfremder Dritter einer gesonderten Anknüpfung bedürften. Die gesellschaftsrechtlichen Schutzzwecke vertragen sich schlecht mit der unmittelbaren ${ }^{28}$ Rechtswahlfreiheit des internationalen Vertragsrechts (Art. 3 Rom I-VO) ${ }^{29}$ Sofern der Gesellschaftsvertrag aber keine Wirkungen für Dritte entfalte, bestehe kein Anlass, die Rechtswahlfreiheit zu begrenzen und die

25 So auch Thomale, NZG 2020, 328, 332.

26 Paulus, in: BeckOGK, Stand: 1.2.2020, Art. 1 Rom I-VO, Rdn.68; Magnus, aaO (Fn. 22), Art. 1 Rom I-VO Rdn. 82.

27 v. Thunen, in: BeckOGK, Stand: 1.10.2018, Internationales Personengesellschaftsrecht, Rdn. 6.

28 Allerdings besteht auch im Gesellschaftsrecht aufgrund der EuGH-Rechtsprechung zur Niederlassungsfreiheit zumindest im Verhältnis zu anderen Mitgliedstaaten eine mittelbare Rechtswahlfreiheit, vgl. hierzu Schwemmer, Anknüpfungsprinzipien im Europäischen Kollisionsrecht, 2018, S. 77 m.w. N.

29 Magnus, aaO (Fn. 22), Art. 1 Rom I-VO Rdn. 79. 
Rechtsbeziehungen dem zwingenden Gesellschaftsstatut zu unterwerfen. ${ }^{30}$ Reine Innengesellschaften, die nach außen nicht als Gesellschaft in Erscheinung treten, sind danach nicht von dem Ausschluss in Art. 1 Abs. 2 lit. f) Rom I-VO erfasst. ${ }^{31}$ Nach dieser Abgrenzung dürfte der Treuhandvertrag einheitlich schuldrechtlich zu qualifizieren sein. Er entfaltet schließlich trotz Begründung einer sog. Quasi-Gesellschafterstellung keine Rechtswirkungen über den Kreis der Treugeber und Gesellschafter hinaus auf gesellschaftsfremde Dritte.

Ob der EuGH diesem im deutschen Schrifttum etablierten Abgrenzungskriterium bei der autonomen Auslegung der Rom I-VO folgen will, ist in der hier besprochenen Entscheidung allerdings offengeblieben. Die Kriterien, die der EuGH bislang als Maßstab angelegt hat, deuten jedoch nicht unbedingt in diese Richtung. Der EuGH fragt nämlich an keiner Stelle nach der Außenwirkung eines Gesellschaftsvertrags, sondern in erster Linie danach, ob Klauseln die „organisatorischen Aspekte“ der Gesellschaft betreffen. ${ }^{32}$ Diesen Grundsatz hatte er bereits in der Entscheidung in der Rechtssache Kerr./.Postnov ${ }^{33}$ aufgestellt. Auch in der hier besprochenen Entscheidung führte der EuGH aus:

„Hierzu hat der Gerichtshof entscheiden, dass der in Art. 1 Abs. 2 Buchst. f der Rom-I-Verordnung vorgesehene Ausschluss von Fragen betreffend das Gesellschaftsrecht, das Vereinsrecht und das Recht der juristischen Personen - wie die Errichtung von Gesellschaften, Vereinen und juristischen Personen durch Eintragung oder auf andere Weise, ibre Rechts- und Handlungsfähigkeit, ibre innere Verfassung und ibre Auflösung - vom Anwendungsbereich der Verordnung ausschließlich die organisatorischen Aspekte dieser Gesellschaften, Vereine und juristischen Personen betrifft. (...) Demnach gilt der Ausschluss dieser Fragen vom Anwendungsbereich (...) für all jene sebr komplexen Rechtsakte, die für die Errichtung einer Gesellschaft erforderlich sind oder ibre innere Verfassung oder ibre Auflösung regeln, d.h. für die unter das Gesellschaftsrecht fallenden Rechtshandlungen." ${ }^{\text {34 }}$

30 Magnus, aaO (Fn. 22), Art. 1 Rom I-VO Rdn. 80; v. Thunen, aaO (Fn. 27), Rdn. 14.

31 Magnus, aaO (Fn. 22), Art. 1 Rom I-VO Rdn. 80; Kieninger, in: Ferrari u.a., Internationales Vertragsrecht, 3. Aufl., 2018, Art. 1 Rom I-VO, Rdn. 2; Paulus, aaO (Fn. 26), Art. 1 Rom I-VO, Rdn. 56; Spickноғ, in: BeckOK BGB, Stand: 1.8.2019, Art. 1 Rom IVO, Rdn. 40; v. Thunen, aaO (Fn. 27), Rdn. 14; Martiny, aaO (Fn. 22), Art. 1 Rom IVO, Rdn. 65; zur Bereichsausnahme in Art. 37 EGBGB a.F. BGH, Urt. v. 13.9.2004, II ZR 276/02, NJW 2004, 3706, 3708; Urt. v. 10.2.2009, VI ZR 28/08, IPRax 2010, 367; Urt. v. 10.6.2015, IV ZR 69/14, NJW 2015, 2581, 2582; SEIBL, IPRax 2010, 347, 351; Hausmann, FS Jayme, Bd. I, 2004, S. 305, 319 (zur Ehegatteninnengesellschaft); a. A. Schall, in: Handkommentar HGB, 3. Aufl., 2020, Anh IntPersGesR Rdn. $30 \mathrm{ff}$; v. Bar/Mankowski, Internationales Privatrecht, Bd. II, 2. Aufl., 2019, 77 Rdn. $129 \mathrm{f}$.

Mit diesem Verständnis auch Thomale, NZG 2020, 328, 332. 
Somit kam es dem EuGH in erster Linie darauf an, ob die jeweiligen Klauseln eine verbandsartige Organisationsstruktur schaffen. Dann wäre aber auch eine reine Innengesellschaft gesellschaftsrechtlich zu qualifizieren, sofern sie Organisationsstrukturen aufweist. ${ }^{35}$

Da der Quasi-Gesellschafter über seine rechtliche Gleichstellung mit den übrigen Gesellschaftern in die Organisationsstruktur der Publikumsgesellschaft einbezogen wird, dürfte seine Rechtsstellung nach den Kriterien des EuGH dem Gesellschaftsstatut zuzuordnen sein. Dies erkennt auch der Generalanwalt Øe in seinen Schlussanträgen. ${ }^{36}$ Bei einer einheitlichen Qualifikation des Vertragswerkes, das diese Quasi-Gesellschafterstellung begründet, spräche danach also viel für eine Zuordnung zum Gesellschaftsstatut. Der EuGH geht diesen Weg der einheitlichen Qualifikation des Vertragswerkes jedoch nicht - im Gegenteil: er will noch nicht einmal den Treuhandvertrag einheitlich qualifizieren.

\section{Verbraucherschützende Sonderanknüpfung}

Selbst wenn man den Treuhandvertrag - ganz oder wie der EuGH in Teilen den Anknüpfungsregeln der Rom I-VO für Schuldverträge unterwirft, ist im unternehmerischen Verkehr ohne Weiteres ein Gleichlauf mit dem Gesellschaftsstatut der Fondsgesellschaft gewährleistet, sei es über eine Rechtswahl (Art. 3 Rom I-VO), sei es über die objektive Anknüpfung an den Sitz des Treuhänders als Dienstleistungserbringer (Art. 4 Abs. 1 lit. b) Rom I-VO). Zu einem Auseinanderfallen von Gesellschaftsstatut und Treuhandvertragsstatut kann es jedoch kommen, wenn die Beteiligungen - wie im Vorlagefall des EuGH - von Verbrauchern im Ausland erworben werden. Denn dann kommt in Ermangelung einer Rechtswahl gemäß Art. 6 Abs. 1 Rom I-VO das Aufenthaltsrecht des Verbrauchers zu Anwendung, soweit der Vertrieb der Beteiligungen auf diesen Aufenthaltsstaat ausgerichtet war.

Etwas anderes würde dann gelten, wenn der Ausnahmetatbestand des Art. 6 Abs. 4 lit. a) Rom I-VO zur Anwendung käme. Dieser nimmt „Verträge über die Erbringung von Dienstleistungen, wenn die dem Verbraucher geschuldeten Dienstleistungen ausschließlich in einem anderen als dem Staat erbracht werden müssen, in dem der Verbraucher seinen gewöhnlichen Aufenthalt hat" von der Sonderanknüpfung aus. Nach Auffassung des EuGH ist dieser Ausnahmetatbestand aber auf Treuhandverträge im Zusammenhang mit Fondsanteilen nicht anwendbar. Er erfasse nur solche Fälle, in denen der Verbraucher keine Möglichkeit habe, die Dienstleistung in seinem Aufenthaltsstaat in Anspruch zu nehmen, sondern sich zu diesem Zweck ins Ausland begeben müsse. 
Hingegen seien Dienstleistungen, die aus der Ferne erbracht werden können, nicht unter die Ausnahme zu fassen. ${ }^{37}$ Es komme darauf an, ob sich schon aus der Natur der Dienstleistung ergebe, dass sie in ihrer Gesamtheit nur im Ausland erbracht werden könne. ${ }^{38}$ Letzteres sah der EuGH als zweifelhaft an, da die Verbraucher die Rechte aus der Treuhandbeteiligung vollständig von ihrem Heimatstaat Österreich aus in Anspruch nehmen konnten. So erhielten sie Geschäftsberichte zugesandt und konnten sich über eine österreichische Webseite informieren sowie ihre Stimmrechte abgeben.

Dieser gegenüber dem recht weit gefassten Wortlaut einschränkenden Auslegung des EuGH ist sowohl aus systematischen als auch aus teleologischen Gründen zuzustimmen. ${ }^{39}$ Als Ausnahmetatbestand ist Art. 6 Abs. 4 Rom I$\mathrm{VO}$ grundsätzlich eng auszulegen, ${ }^{40}$ zumal sich dort die Interessen bestimmter Mitgliedsstaaten und Wirtschaftszweige niedergeschlagen haben - im Falle des Art. 6 Abs. 4 lit. a Rom I-VO diejenigen der Tourismusindustrie. ${ }^{41}$ So nennt denn auch der Giuliano/Lagarde-Bericht als Anwendungsbeispiel für die Vorgängervorschrift des EVÜ nur im Reiseland erbrachte touristische Dienstleistungen wie Sprachkurse oder Hotelübernachtungen. ${ }^{42}$ Die Schutzbedürftigkeit des Verbrauchers tritt aber erst dann gegenüber den Interessen des Dienstleisters zurück, wenn er sich zur Inanspruchnahme der Dienstleistung in das Ausland zum Sitz des Dienstleisters begibt. Bei Dienstleistungen, die ihm (digital oder postalisch) an seinem Wohnsitz erbracht werden, soll der Verbraucher sich auf seinen heimischen Verbraucherschutzstandard verlassen dürfen.

\section{Rechtsmissbräuchlichkeit der Rechtswablklausel}

Schließlich hatte der EuGH die Frage zu beantworten, ob die - gemäß Art. 6 Abs. 2 Rom I-VO auch bei Verbraucherverträgen grundsätzlich zulässige, aber in ihren Rechtswirkungen beschränkte - Rechtswahlklausel zugunsten des deutschen Rechts rechtsmissbräuchlich im Sinne des Art. 3 Abs. 1 der Klausel-

37 EuGH, Urt. v. 3.10.2019, Rs. C-272/18 - VKI./.TVP, Rdn. 54.

38 EuGH, Urt. v. 3.10.2019, Rs. C-272/18 - VKI./.TVP, Rdn. $51 \mathrm{f}$.

39 So auch Mankowski, LMK 2019, 422737; Reuter, WuB 2020, 107, 110. In der Literatur wurde eine solche einschränkende Auslegung bereits seit längerem diskutiert, vgl. MARTiny, aaO (Fn. 22), Art. 6 Rom I-VO. Zu Art. 29 EGBGB a.F. bereits Mankowski, RabelsZ 63 (1999), 203, 254 f; SPINDLER, IPRax 2001, 400, 408.

40 RÜHL, in: BeckOGK, Stand: 1.7.2019, Art. 6 Rom I-VO, Rdn. 116 m. w. N.

41 Vgl. GA ØE, Schlussanträge v. 5. 9. 2019, Rs. C-272/19 - VKI./.TVP, Rdn. 74 ff; MANKOwSKI, Interessenpolitik und europäisches Kollisionsrecht, 2011, S. 48f; DERs., LMK 2019, 422737.

42 Bericht Giuliano/Lagarde, BT-Drucks. 10/503, S. 36, 57. 
richtlinie 93/13 EWG ${ }^{43}$ ist. Dies bejahte der EuGH. Der Verbraucher werde durch eine in AGB enthaltene Rechtswahlklausel in die Irre geführt, weil sie suggeriere, dass der Vertrag allein und ausschließlich dem gewählten Recht unterliege. Der Verwender müsse den Verbraucher deshalb darauf hinweisen, dass er gemäß Art. 6 Abs. 2 der Rom I-VO trotz wirksamer Rechtwahl jedenfalls den Schutz der zwingenden Vorschriften seines Aufenthaltsrechts genießt. ${ }^{44}$ Diesen Grundsatz hatte der EuGH bereits in seiner Entscheidung in der Rechtssache VKI./.Amazon für im elektronischen Geschäftsverkehr geschlossene Verträge aufgestellt. ${ }^{45}$ Er bestätigt an dieser Stelle lediglich seine damalige Entscheidung und erklärt sie für alle Rechtswahlklauseln in AGB für anwendbar. ${ }^{46}$ Diese Rechtsprechung hat bereits einige Aufmerksamkeit in der Literatur erfahren. ${ }^{47}$ Sie wurde dabei zum Teil als systemwidrig kritisiert. ${ }^{48}$ In der Tat besteht der Gedanke des Art. 6 Abs. 2 Rom I-VO gerade darin, dem Verbraucher durch den Günstigkeitsvergleich die Vorteile sowohl seiner Heimatrechtsordnung als auch des gewählten Rechts zu sichern. Bei einer Unwirksamkeit der Rechtswahl steht der Verbraucher somit materiellrechtlich nicht unbedingt besser da. ${ }^{49} \mathrm{Da}$ die ganz überwiegende Mehrheit der Verbraucher aber die Rechtswahlklausel nicht hinterfragt, ist der Günstigkeitsvergleich meist völlig wirkungslos. Mit der Transparenzkontrolle mag der EuGH bei aller methodischen Kritik ein Instrument gefunden haben, dem Günstigkeitsvergleich zu praktischer Wirksamkeit zu verhelfen.

\section{Konsequenzen der Entscheidung für bestehende Treubandbeteiligungen}

Der EuGH stärkt mit seiner Entscheidung den kollisionsrechtlichen Verbraucherschutz als eines der politischen Ziele des vereinheitlichten europäischen IPR. ${ }^{50} \mathrm{Ob}$ sich dies aber im Falle von Treuhandbeteiligungen auch im Ergebnis der Rechtsanwendung immer im Sinne des Verbraucherschutzes auswirken wird, ist damit noch nicht gesagt. Jedenfalls schafft der EuGH mit seiner Ent-

43 Richtlinie 93/13/EWG des Rates vom 5. April 1993 über missbräuchliche Klauseln in Verbraucherverträgen, ABl. EG Nr. L 95/29.

44 EuGH, Urt. v. 3.10.2019, Rs. C-272/18 - VKI./.TVP, Rdn. $58 \mathrm{f}$.

45 EuGH, Urt. v. 28.7.2016, Rs. C-191/15 - VKI./.Amazon EU Sàrl, Rdn. 71.

46 Vgl. hierzu auch ManKowsKi, LMK 2019, 422737.

47 Vgl. z.B. Mankowski, NJW 2016, 2705; Ders., IPRax 2019, 208 m.w.N; Rühl, aaO (Fn. 40), Art. 6 Rom I-VO, Rdn. 249 ff; DIES., C.M.L.Rev. 55 (2018), 201 ff; RiELÄNDER, RIW 2017, 28; Rотт, EuZW 2016, 733, 736.

48 Mankowski, NJW 2016, 2705, 2706f; Rühl, aaO (Fn. 40), Art. 6 Rom I-VO, Rdn. 251; DIES., C.M.L.Rev. 55 (2018), 201, $209 f$.

49 Rühl, aaO (Fn. 40), Art. 6 Rom I-VO, Rdn. 249 ff; Mankowski, NJW 2016, 2705; KloCKE, DZWIR 2020, 61, 64.

50 Vgl. Schwemmer, aaO (Fn. 28), S. 139 ff, 201. 
scheidung erhebliche Rechtsunsicherheit für bestehende Treuhandbeteiligungen.

\section{Rechtsunsicherheit über das auf den Treubandvertrag anwendbare Recht}

Während sich die Gründungsgesellschafter bei der Strukturierung neuer Treuhandbeteiligungen durch die Formulierung entsprechend transparenter Rechtswahlklauseln auf die neuen Anforderungen einstellen können, dürfte bei bestehenden Treuhandbeteiligungen kaum ein Vertragswerk eine nach den Kriterien des EuGH wirksame Rechtswahlklausel enthalten. Diese Rechtswahlklauseln stellten aber jedenfalls Klarheit hinsichtlich des anwendbaren Rechts her. Der Treuhandvertrag unterlag in jedem Fall dem Recht der Fondsgesellschaft, sei es über die Rechtswahlklausel bei Einordnung als Schuldvertrag oder sei es qua objektiver, zwingender Anknüpfung bei gesellschaftsrechtlicher Qualifikation. Ist die Rechtswahl nun unwirksam, kommt es hingegen auf die Qualifikationsfrage entscheidend an.

Da der EuGH es auf der anderen Seite vermieden hat, diese Frage für den Treuhandvertrag umfassend und einheitlich zu beantworten, besteht für bestehende Treuhandbeteiligungen nun erhebliche Rechtsunsicherheit. Es ist nach der Entscheidung des EuGH weitgehend unklar, ob der Treuhandvertrag nun in seiner Gänze als Verbrauchervertrag im Sinne des Art. 6 Rom I-VO dem Heimatrecht des Anlegers unterliegt, oder ob dies entsprechend der Logik der gespaltenen Anknüpfung nur für Teile dieses Vertrag - und wenn ja, für welche - gilt.

\section{Friktionen zwischen Gesellschafts- und Treuhandvertragsstatut}

Klar ist nach der Entscheidung des EuGH aber jedenfalls, dass das eng verzahnte Vertragswerk aus Treuhand- und Gesellschaftsvertrag nicht einheitlich gesellschaftsrechtlich zu qualifizieren ist. Dies wird unweigerlich zu Friktionen bei der Rechtsanwendung führen. Besonders relevant wird dies, wenn der Verbraucher sich aufgrund einer (teilweise) vertragsrechtlichen Qualifikation des Treuhandvertrags nach den Regelungen seines Heimatrechts durch Rücktritt, Anfechtung oder Widerruf ex tunc von seiner Beteiligung lösen will. Das deutsche Gesellschaftsstatut sieht nach der Lehre von der fehlerhaften Gesellschaft nur eine Kündigung mit Wirkung ex nunc vor; dies gilt auch für den Quasi-Gesellschafter. ${ }^{51}$ Gegebenenfalls hätte also die Treuhandkommanditistin den Treuhandvertrag mit dem ausländischen Anleger vollständig rückabzu- 
wickeln, während nach dem Gesellschaftsstatut nur eine Auszahlung des Abfindungsguthabens in Betracht kommt. Das IPR hält für solche Fälle zwar mit dem Rechtsinstitut der Anpassung ${ }^{52}$ eine Möglichkeit bereit, zu einer angemessenen Lösung zu kommen. Sachgerechter wäre es jedoch, die dépeçage des einheitlichen Vertragswerkes von vornherein zu vermeiden.

\section{Zusammenfassende Thesen}

1. Der EuGH nimmt in seiner Entscheidung nicht umfassend zur kollisionsrechtlichen Behandlung von Treuhandverträgen über Fondsanteile Stellung. Vielmehr nimmt er nur diejenigen Klauseln in den Blick, die allein die Leistungserbringung zwischen Treuhänderin und Treugeber regeln. Diese sollen als schuldvertragliche Regelungen der Rom I-VO unterfallen. Auf die Einordnung derjenigen Klauseln, die die Rechtsstellung des Treugebers in der Fondsgesellschaft betreffen, komme es insoweit nicht an.

2. Der methodische Ansatz des EuGH führt regelmäßig zu einer gespaltenen Anknüpfung von Gesellschaftsverträgen. Dies widerspricht den Grundsätzen der Rom I-VO, die Verträge einem einheitlichen Statut unterwerfen will. Darüber hinaus führt die kollisionsrechtliche dépeçage von Gesellschaftsverträgen zwangsläufig zu Anpassungsproblemen in der Rechtsanwendung.

3. Es spricht einiges dafür, dass der EuGH solche Klauseln, die die Rechtsstellung des Treuhänders als Quasi-Gesellschafter regeln, dem Gesellschaftsstatut zuordnen würde, da sie die organisatorischen Aspekte der Gesellschaft betreffen. Es zeichnet sich insoweit eine neue Grenzziehung zwischen dem Schuldvertragsrecht und dem Gesellschaftsrecht ab. Während in der deutschen Literatur bislang in erster Linie danach gefragt wird, ob der Vertrag Rechtswirkungen nach außen auf gesellschaftsfremde Dritte entfaltet, stellt der EuGH vor allem darauf ab, ob eine verbandsartige Organisationsstruktur besteht.

4. Soweit der Treuhandvertrag als Schuldvertrag unter die Rom I-VO fällt, kommt gegenüber Verbrauchern die Sonderanknüpfung des Art. 6 Rom I-VO zur Anwendung. Die Ausnahmevorschrift des Art. 6 Abs. 4 lit. a) Rom I-VO greift nicht, da der Verbraucher die Dienstleistung von seinem Aufenthaltsstaat aus in Anspruch nehmen kann.

5. Der EuGH bestätigt seine Rechtsprechung, wonach in AGB enthaltene Rechtswahlklauseln gegenüber Verbrauchern rechtsmissbräuchlich und damit unwirksam sind, wenn der Verwender den Verbraucher nicht darauf

52 Vgl. zu dieser Methode des IPR nur Kropholler, Internationales Privatrecht, 5. Aufl., 2004, \34. 
hinweist, dass er gemäß Art. 6 Abs. 2 der Rom I-VO jedenfalls den Schutz der zwingenden Vorschriften seines Aufenthaltsrechts genießt. 
